\title{
Two-stage current transformer with electronic compensation
}

\author{
Daniel Slomovitz, Leonardo Trigo, Carlos Faverio
}

UTE - Laboratory, Paraguay 2385, Montevideo, Uruguay

A current transformer with electronic assistance was developed, intended for current to voltage transducers. It is based on the twostage principle, and it includes electronic compensation for magnetizing errors as well as capacitive errors. The nominal input currents go from $1 \mathrm{~A}$ up to $100 \mathrm{~A}$, and the nominal output current is $0.2 \mathrm{~A}$. Using a load of $10 \Omega$, the nominal output voltage is $2 \mathrm{~V}$, with $0.4 \mathrm{~W}$ of dissipation.

Keywords: Current transformer; compensation; measurement; calibration; power; capacitance; two-stages.

Citation: Daniel Slomovitz, Leonardo Trigo, Carlos Faverio, Two-stage current transformer with electronic compensation, Acta IMEKO, vol. 1, no. 1, article 16, July 2012, identifier: IMEKO-ACTA-01(2012)-01-16

Editor: Pedro Ramos, Instituto de Telecomunicações and Instituto Superior Técnico/Universidade Técnica de Lisboa, Portugal

Received January $20^{\text {th }}, 2012$; In final form June $8^{\text {th }}, 2012$; Published July 2012

Copyright: (C) 2012 IMEKO. This is an open-access article distributed under the terms of the Creative Commons Attribution 3.0 License, which permits unrestricted use, distribution, and reproduction in any medium, provided the original author and source are credited

Funding: No information provided

Corresponding author: Daniel Slomovitz, e-mail: dslomo@gmail.com

\section{INTRODUCTION}

Very high precision wattmeters have been developed in National Metrology Institutes. Some of them are based on adding devices [1], others on bridges [2], and others use two commercial digital sampling voltmeters (DSV), like the model HP $3458[3,4]$. Most of them require a current-to-voltage transducer for the input current. If a simple resistor shunt is used as this transducer, the maximum current is limited by the maximum allowed dissipation. It is not possible to get very low uncertainties if the currents are higher than some miliamperes. This leads to the use of current transformers (CT) to reduce the current through the shunt to a small value, so that the shunt dissipates a small power with low temperature rise. Burdens around $10 \Omega$ for output voltages between $1 \mathrm{~V}$ and $2 \mathrm{~V}$ lead to powers lower than $1 \mathrm{~W}$, which are acceptable for this kind of application.

When two DSVs are used, one is used to digitize the voltage and the other a voltage proportional to the current. As the best voltage range of the mentioned instrument is $10 \mathrm{~V}$, it is necessary to scale currents that can reach up to $100 \mathrm{~A}$ to voltages of few volts. The uncertainty of this model of DSV, using special control programs $[5,6]$, is in the order of some parts in $10^{6}$. Then, the whole current to voltage transducer must have uncertainties around of a few parts in $10^{6}$, in order not to degrade the system. As this transducer comprises a resistor shunt and a CT, this last device must have uncertainties around 1 part in $10^{6}$. To get that, a two-stage transformer $[7,8]$ is proposed, but this proposal adds electronic compensations. Although electronic compensation of two-stage transformers have been proposed in the past [9-18], none of them reaches a complete compensation of the internal transformer error sources. They do not compensate errors caused by internal resistance of the windings and errors coming from internal stray capacitances.

For this type of CT, the main source of error is the magnetizing current $\left(I_{\mathrm{m}}\right)$. This current flows through the magnetizing branch, so that it does not flow through the output. Figure 1 shows a schematic model of this type of transformer. $Z_{1}$ and $Z_{2}$ are the series impedances with resistive and inductive components $\left(R_{2}, L_{2}\right)$, while $R_{m}, L_{m}$ represent the components of the magnetizing branch. $C_{1}, C_{2}$ represent stray capacitances in the primary and secondary windings.

There is no capacitance between primary and secondary due to electrostatic shields that this kind of CT has. To get low errors, the magnetic flux must be as low as possible, reducing in

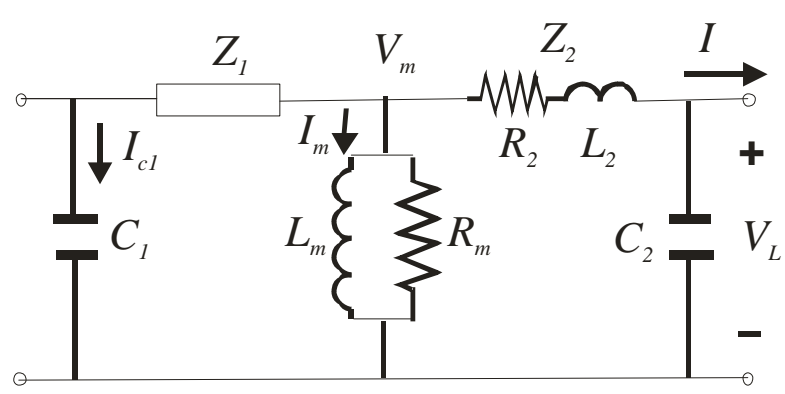

Figure 1. Transformer model. 
this way the magnetizing current. For that purpose, the magnetizing voltage $\left(V_{m}\right)$ must be reduced as much as possible. With conventional CTs there is a limit due to the voltage drop on the load, which in our case is very high, $2 \mathrm{~V}$ at $0.2 \mathrm{~A}$. Usually, standard CT loads are around $0.2 \Omega$, that is 50 times lower. To reach lower errors, two-stage CTs are used in this application. In two-stage transformers [7], one stage provides the power needed for the core magnetization and for the burden (magnetizing core), while the other stage only supplies the error current of the first stage (ratio core), being its magnetic flux very low but not zero. The exact value of this flux depends on the external burden and the internal series impedance of its secondary winding. The lower the load is, the smaller the magnetic flux. This method requires one to add the currents of both secondary windings, what is done using different methods depending on the application. In current to voltage transducer, this addition can be done using two burden resistors instead of only one [19]. One resistor, of high precision, is the burden of the magnetizing secondary winding and the other one, generally of the same value but lower precision, is the burden of the ratio winding (see Figure 2). $W_{\mathrm{m}}$ is the magnetizing winding of the two-stage transformer and $W_{\mathrm{c}}$ is the ratio one. The core to the left, in Figure 2, is the magnetizing one, and the core to the right is the ratio one. As the compensating current through $W_{\mathrm{c}}$ is much smaller than the main one (through $W_{\mathrm{m}}$ ), the same occurs to the voltage drops on $R_{c}$ and $R_{m}$, reducing the magnetization current of the compensating core. This allows total errors in the order of 10 parts in $10^{6}$ if burdens around $10 \Omega$ are used [19]. For reducing the errors 10 times more, as it is the goal of this proposal, an electronic compensating circuit is added.

\section{TRANSFORMER DESIGN}

The core for both stages is of a high permeability type (Mumetal). Both secondary windings (magnetizing and ratio stages) have 500 turns each, as Figure 3 shows. There are two groups of primary windings. The first one is formed by 10 groups of 10 turns each. Connecting in parallel-series sets, it can be arranged from 10 to 100 effective turns $(10,20,50$ and 100). As the nominal secondary current is $200 \mathrm{~mA}$, the nominal input current ranges are $1 \mathrm{~A}, 2 \mathrm{~A}, 5 \mathrm{~A}$ and $10 \mathrm{~A}$. For the upper ranges, another primary winding group exists. It has 10 groups of 1 turn each. Therefore, using these winding groups, the nominal primary current goes from $10 \mathrm{~A}$ up to $100 \mathrm{~A}$. Table 1 shows the different ratios available. Each group of turns in each winding has the same current whichever were the connection. In this way, the stray magnetic flux does not change when different ratios are selected. This property ensures that the errors are practically the same for all ratios, allowing a reduction in the calibration work. However, error differences can appear between low and high current windings, because they have different turns. To determine the amount of this variation,

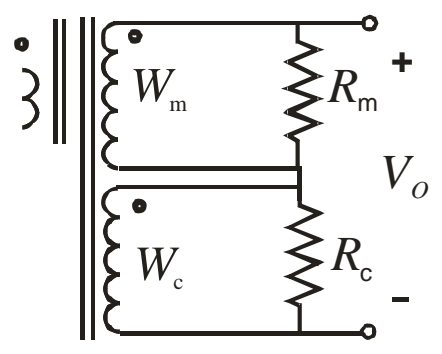

Figure 2. Conventional two-stage current to voltage transducer.

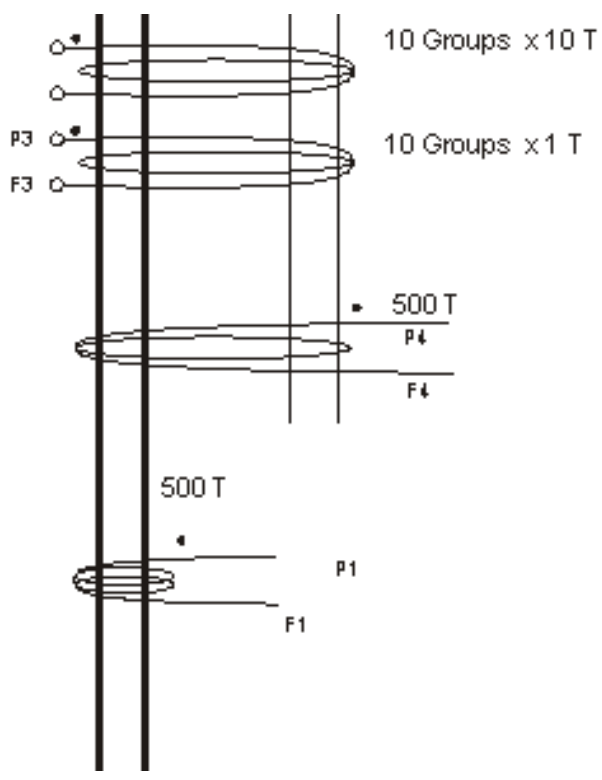

Figure 3 . Windings of the proposed transformer. Compensating core to the left, main core to the right.

there is a $10 \mathrm{~A}$ range in each winding. Connecting both with opposite polarity, ideally, the output must be null. Then, measuring the actual output, that ratio error difference can be calculated.

\section{ELECTRONIC COMPENSATION}

The goal of the compensating circuit is to null the magnetic flux in the compensating stage core. For that, a controlled voltage source generates the same value as the sum of the voltage drops in the internal and external series impedances of the compensating secondary winding, but with opposite polarity. In this way, no magnetizing voltage exists in this winding, neither magnetizing current $I$. From a theoretic point of view, there is a complete compensation and null errors. This electronic source supplies only the small power required by the compensating stage of the transformer, so that a simple operational amplifier is enough for this application. Figure 4 shows a schematic circuit.

The purpose of the electronic device is to null the electromotive force (emf) $\varepsilon$ in $\mathrm{W}_{\mathrm{c}}$. Assuming an internal resistive impedance $r$ of this winding, the following equations apply:

$\varepsilon=I\left(r+R_{1}\right)+V_{2}$

and

Table 1. Available ratios using different series-parallel connections.

\begin{tabular}{ccccc}
\hline $\begin{array}{c}\text { Parallel } \\
\text { groups }\end{array}$ & Groups & Turn/Group & Ratio & $\begin{array}{c}\text { Input } \\
\text { Current (A) }\end{array}$ \\
\hline 1 & 10 & 10 & 5 & 1 \\
2 & 5 & 10 & 10 & 2 \\
5 & 2 & 10 & 25 & 5 \\
10 & 1 & 10 & 50 & 10 \\
\hline 1 & 10 & 1 & 50 & 10 \\
2 & 5 & 1 & 100 & 20 \\
5 & 2 & 1 & 250 & 50 \\
10 & 1 & 1 & 500 & 100 \\
\hline
\end{tabular}




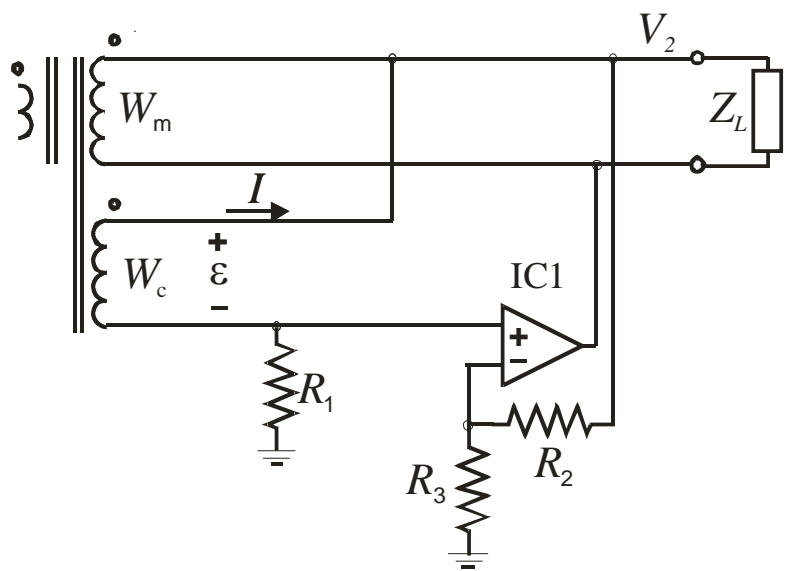

Figure 4. Schematic circuit of the two-stage electronic compensated transformer.

$V_{2}=-R_{1} I\left(1+\frac{R_{2}}{R_{3}}\right)$,

which leads to

$$
\varepsilon=I\left(r-\frac{\mathrm{R}_{1} \mathrm{R}_{2}}{\mathrm{R}_{3}}\right) .
$$

If $r=R_{1} R_{2} / R_{3}$, then $\varepsilon=0$. This shows that it is possible to null the emf in $W_{c}$, nulling in this way the magnetic flux through the auxiliary core.

\section{ERROR SOURCES}

As mentioned, the first error source is the magnetizing current. In the prototype, it was reduced 100 times with the electronic compensator, reaching errors in the order of $0.1 \mu \mathrm{A} / \mathrm{A}$ (in phase and in quadrature). This value includes ambient temperature variation. The influence of the latter on the compensation is due to the variation of the resistance of the windings, that vary around $0.4 \% / \mathrm{K}$, affecting equation (3).

Second order errors include stray capacitances and stray magnetic fluxes. For the first ones, an electrostatic shield between primary and secondary windings and an electronic compensation to reduce the influence of some stray capacitances were used. The shield was connected to an external guard terminal. In this way, stray capacitances between primary and secondary windings are eliminated. However, stray capacitances between all windings and shields remain. It is

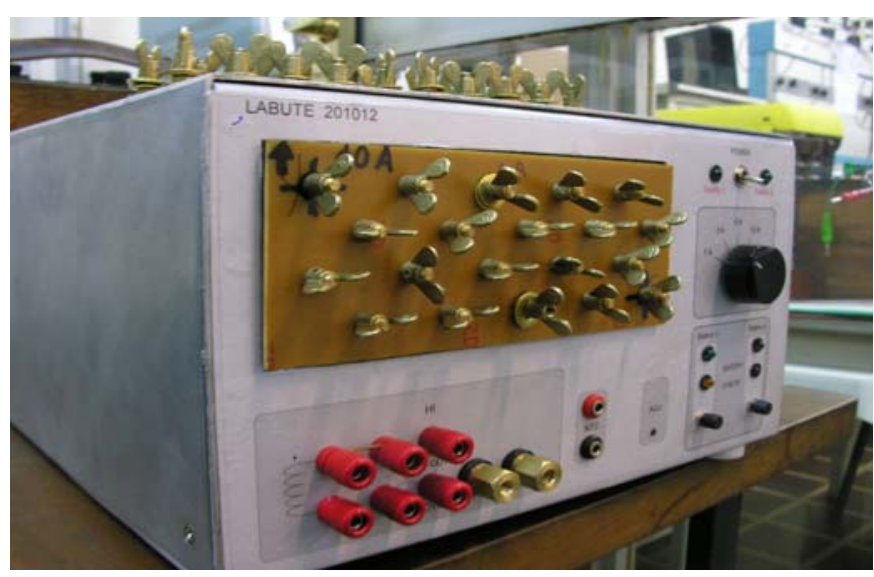

Figure 5. Prototype of the proposed transformer.
Table 2. Calibration of the proposed transformer.

\begin{tabular}{cccc}
\hline Ratio & $\begin{array}{c}\text { Primary current } \\
\text { (A) }\end{array}$ & $\begin{array}{c}\text { Error in phase } \\
\mathbf{\times 1 0 ^ { - 6 }}\end{array}$ & $\begin{array}{c}\text { Error in quadrature } \\
\mathbf{\times 1 0 ^ { - 6 }}\end{array}$ \\
\hline 10 & 2 & -0.6 & -0.8 \\
5 & 1 & -0.4 & -0.6 \\
\hline
\end{tabular}

possible to reduce this effect using shielded cables for the windings [20], but this technique was not used because of the high cable section required in this design. In the secondaries, these capacitances produce errors in quadrature that only depends on the resistive burden. With $10 \Omega$ burden, as is used, this error source cannot be neglected. To compensate it, an electronic device that simulates a negative capacitor was included at the output, in parallel with the burden. Its value was adjusted in such way that no error differences exist when the burden was changed between $0 \Omega$ and $10 \Omega$.

Stray capacitances between primary windings themselves, and to shield, changes when different series-parallel connections are used. However, all these capacitances have low values due to the small number of turns that these windings have, leading to errors lower than 1 part in $10^{6}$.

To reduce the effect of stray magnetic fluxes and magnetic shields an equalization winding was added. This winding has 5 partial windings of 5 turns, all connected in parallel, around the core. In this way, it forces the flux to be homogeneous, reducing this error source.

The influence of these second order errors in the whole performance was estimated in $0.4 \mu \mathrm{A} / \mathrm{A}$ for the prototype. Then, the total error, including the magnetizing current error, is $0.5 \mu \mathrm{A} / \mathrm{A}$, approximately.

To confirm this theoretical error estimation, the prototype was tested against a current comparator, with uncertainties of $0.3 \mu \mathrm{A} / \mathrm{A}$ (in phase and in quadrature). The tested ratios were 5 and 10 , and the results are shown in Table 2.

The uncertainty of this calibration is mainly due to the uncertainty of the current comparator, including its zero detector, previously stated $(0.3 \mu \mathrm{A} / \mathrm{A})$. The error values are basically covered by the estimated errors of the prototype and the uncertainty of the calibration, confirming the theoretic analysis.

\section{PROTOTYPE}

Figure 5 shows a photo of the prototype. It has two output binding posts (vertical, red at the left) corresponding to the transformer secondary. The four red binding posts in the middle corresponds to the burden resistor, and the two metallic ones, to guard and ground. The banana couple black and red are connected to a thermistor included in the burden resistor to measure its temperature. Other switches and LEDs, at the right, correspond to power supplies. To eliminate interference from power network, batteries are used.

At the front panel, it has 10 pairs of terminals of low-current winding (10 groups of 10 turns). Each group is connected to each vertical pair of terminals. The combinations in seriesparallel connections are made by changing a circuit printed board (PCB) that is connected at the front of the case. The cooper side of this PCB is on the inner face of the case, so it is not visible. Figure 6 shows two PCBs on their cooper sides for $1 \mathrm{~A}$ and $5 \mathrm{~A}$. The upper one connects all winding groups in series, forming a winding of 100 turns. The lower PCB connects the first five groups in parallel, in series with the last 

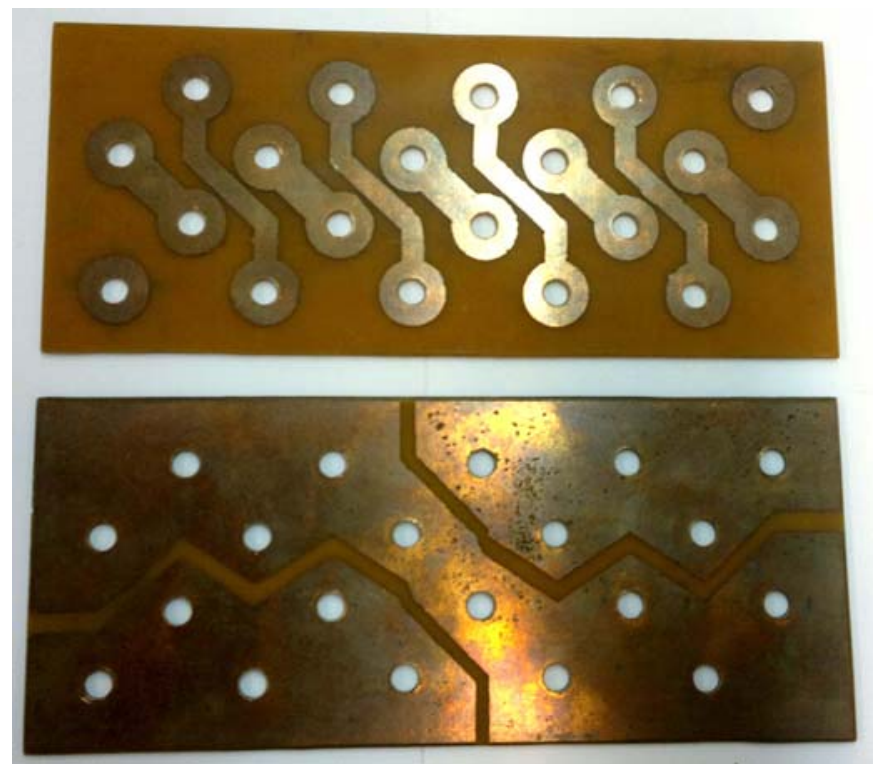

Figure 6. Circuit printed boards used to connect the groups of low-current primary windings. The upper one corresponds to $1 \mathrm{~A}$ of nominal current (100 turns), and the lower to $5 \mathrm{~A}$ (20 turns).

five groups. In this way, the effective number of turns is 20 . There is one printed board for each ratio, with different connections. For $10 \mathrm{~A}$, all groups are connected in parallel.

A similar system exists for the high-current winding (10 groups of 1 turn) in the upper side of the case (see Figure 7). It also has 10 pairs of terminals that can be connected in different series-parallel configurations. As the currents in these windings are higher than the first ones, the connections are made by using copper bars instead of PCBs.

\section{CONCLUSIONS}

A current transformer intended for high precision currentto-voltage transducers was proposed. It uses a two-stage technique plus electronic assistance. This last device cancels the magnetic flux in the compensating core, canceling the error of the transformer due to this cause. It does not depend on the internal winding resistance or the external burden. The primary

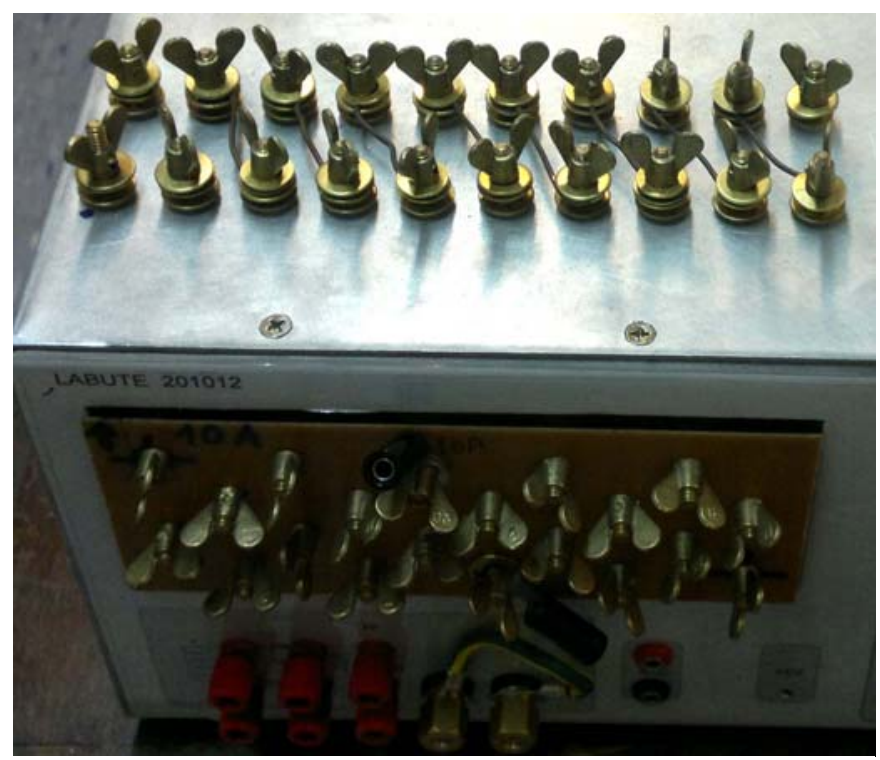

Figure 7. Connection terminals for high-current windings at the upper side of the case. windings can be arranged in different series-parallel connections covering ranges from $1 \mathrm{~A}$ to $100 \mathrm{~A}$. The estimated ratio error is about $0.5 \mu \mathrm{A} / \mathrm{A}$.

\section{REFERENCES}

[1] P. Braga, D. Slomovitz, "RMS Voltmeter Based Power and Power-Factor Measuring System," International Journal of Electronics, vol. 75, no 3, Set. 1993, pp. 561-565.

[2] J. Bai, L. Yang, S. Zhao, J. Zong, E. So, "The Establishment of Power/Energy Standard at China Electric Power Research Institute and its Comparison with NRC," Conference on Precision Electromagnetic Measurements, Conference Digest, June 2010, pp. 275-276.

[3] E. Tóth, A. M. Ribeiro Franco, and R. M. Debatin, "Power and Energy Reference System, Applying Dual-Channel Sampling," IEEE Trans. Instrum. Meas., vol. 54, no. 1, Feb. 2005, pp. 404-408.

[4] U. Pogliano, "Use of Integrative Analog-to-Digital Converters for High-Precision Measurement of Electrical Power," IEEE Trans. Instrum. Meas., vol. 50, no. 5, Oct. 2001, pp. 1315-1318.

[5] R. L. Swerlein, "A $10 \mathrm{ppm}$ Accurate Digital AC Measurement Algorithm,” Proc. NCSL, Albuquerque, USA, Aug. 1991, pp. 17-36.

[6] G.A. Kyriazis, R. Swerlein, "Evaluation of Uncertainty in AC Voltage Measurement Using a Digital Voltmeter and Swerlein's Algorithm," Conference on Precision Electromagnetic Measurements, Conference Digest, June 2002, pp. 24-25.

[7] H. B. Brooks, F. C. Holtz, "The two-stage current transformer," AIEE Trans., vol. 41, June 1922, pp. 382-393.

[8] P. J. Betts, "Two-stage current transformers in differential calibration circuits," IEE Proc., vol. 130, Pt. A, no. 6, Sept. 1983, pp. 324-328.

[9] D. L. H. Gibbibngs, "A circuit for reducing the exciting current of inductive devices," Proc. IEE, vol. 108,1961, pp. 339-349.

[10] O. Peterson, "A self-balancing current comparator," IEEE Transs on Instru. and Meas., vol. IM-15, 1966, pp. 62-71.

[11] R. Friedl, "Current transformers with electronic error compensation," Messkechnik, vol. 76, no 10, 1968, pp. 241-250.

[12] T. M. Souders, "Wide-band two-stage current transformer of high accuracy," IEEE Trans. Instrum. Meas., vol. 4, June 1972, pp. 340-349.

[13] G. E. Bear, "100:1 step-up amplifier-aided two-stage current transformers with small error at $60 \mathrm{~Hz}$," IEEE Trans. Instrum. Meas., vol. 28, June 1979, pp. 146-152.

[14] P. N. Miljanic, E. So, W. J. M Moore, “An electronically enhanced magnetic core for current transformers," Conference on Precision Electromagnetic Measurements, June 1990, pp. 328.

[15] J. L. West, P. N. Miljanic, "An improved two-stage current transformer,” IEEE Trans. Instrum. Meas., vol. 40, June 1991, pp. 633-635.

[16] E. So, S. Ren, D.A. Bennett, "High-current high-precision openable-core AC and AC/DC current transformers," IEEE Trans. on Instrum. and Meas., vol. 42, no. 2, April 1993, pp. 571-576.

[17] E. So, D. A. Bennett, "A low-current multistage clamp-on current transformer with errors below $50 \times 10^{-6}$," IEEE Trans. Instrum. Meas., vol. 46, Apr. 1997, pp. 454-458.

[18] J. L. West, P. N. Miljanic, "An improved two-stage current transformer," IEEE Trans. Instrum. Meas., vol. 40, June 1991, pp. 633-635.

[19] CONIMED, Nota de Aplicación - Modelo CCT20, Conversor Corriente Tensión de Gran Exactitud, www.conimed.com.

[20] D. Slomovitz, H. de Souza, "Shielded Electronic Current Transformer," IEEE Trans. Instrum. Meas., vol. 54, no. 2, Apr. 2005, pp. 500-502. 